\title{
Emergence of Constructor-Based Irreversibility in Quantum Systems: Theory and Experiment
}

\author{
Chiara Marletto and Vlatko Vedral \\ Clarendon Laboratory, University of Oxford, Parks Road, Oxford OX1 3PU, United Kingdom \\ and Fondazione ISI, Via Chisola 5, Torino, Italy \\ and Centre for Quantum Technologies, National University of Singapore, 3 Science Drive 2, Singapore 117543 \\ and Department of Physics, National University of Singapore, 2 Science Drive 3, Singapore 117542 \\ Laura T. Knollø, Fabrizio Piacentini®, Ettore Bernardi®, Enrico Rebufello®, \\ Alessio Avella, and Marco Gramegna@ \\ Istituto Nazionale di Ricerca Metrologica, Strada delle Cacce 91, 10135 Torino, Italy \\ Ivo Pietro Degiovanni and Marco Genovese 10 \\ Istituto Nazionale di Ricerca Metrologica, Strada delle Cacce 91, 10135 Torino, Italy \\ and INFN, sezione di Torino, via P. Giuria 1, 10125 Torino, Italy
}

(Received 18 June 2021; accepted 14 January 2022; published 23 February 2022)

\begin{abstract}
How irreversibility arises in a universe with time-reversal symmetric laws is a central problem in physics. In this Letter, we discuss a radically different take on the emergence of irreversibility, adopting the recently proposed constructor theory framework. Irreversibility is expressed as the requirement that a task is possible, while its inverse is not. We prove the compatibility of such irreversibility with quantum theory's time-reversal symmetric laws, using a dynamical model based on the universal quantum homogenizer. We also test the physical realizability of this model by means of an experimental demonstration with highquality single-photon qubits.
\end{abstract}

DOI: $10.1103 /$ PhysRevLett.128.080401

Introduction.-The emergence of irreversibility from time-symmetric physical laws is a central problem in contemporary physics. Indeed, there are several approaches to irreversibility in physics: statistical mechanics methods [1-3]; information-theoretic descriptions of logically irreversible tasks [4-6]; classical and quantum thermodynamics second laws [2,7-9]. In all such cases, a tension arises between the laws describing irreversible phenomena, and the time-reversal symmetry of microscopic dynamics.

In this Letter, we express irreversibility as the requirement that a transformation is possible (i.e., it can be realized limitlessly well by a system operating in a cycle), while its inverse is not. The origin of this irreversibility can be intuitively understood considering Joule's experiment [2]: while a volume of water can be heated up by mechanical means only, it is impossible to cool it down by the same means. More generally, if a transformation can be realized arbitrarily well by a machine working in a cycle, the same might not hold for the reverse transformation, even under

Published by the American Physical Society under the terms of the Creative Commons Attribution 4.0 International license. Further distribution of this work must maintain attribution to the author(s) and the published article's title, journal citation, and DOI. time-reversal symmetric laws. The concept of a cycle performing a transformation, central to thermodynamics, was generalized by von Neumann to a constructor-a system able to perform a given task on another system while retaining the ability to do it again. Therefore, we call the generalization of Joule's experiment irreversibility "constructor-based irreversibility." To analyze it formally, we use constructor theory, a recently proposed extension of quantum computation to cover general tasks [10,11]. Compared with traditional approaches to irreversibility in quantum and classical thermodynamics, this one presents many advantages. Unlike the usual information-theoretic description, it does not suffer from the circularity between "information" and "distinguishability" definitions [11]. In contrast with quantum or classical statistical mechanics approaches [12], it does not rely on dynamical trajectories in phase space (difficult to define in quantum theory), on statistical approximations such as coarse graining or typicality arguments. With respect to quantum thermodynamics, it overcomes the issue related to the destruction of quantum coherences between system and reservoir [13] related to the two-point measurement scheme definition of work [14].

Besides, we illustrate how, surprisingly, within a qubitbased toy model this irreversibility is compatible with 
quantum theory time-reversal symmetric laws. We also provide a quantitative test of this claim by means of a quantum optics experiment.

Consider a universe made of infinite qubits, available in any state, where all unitary transformations and their transposes are allowed. A task $T$ is the specification of a physical transformation on qubits, e.g., from a quantum state $\rho_{x}$ to another one $\rho_{y}$ :

$$
T=\left\{\rho_{x} \rightarrow \rho_{y}\right\},
$$

whose transpose $T^{\sim}$ is defined as

$$
T^{\sim}=\left\{\rho_{y} \rightarrow \rho_{x}\right\} .
$$

We will label the substrate qubit on which $T$ is defined as $Q$, and the rest of the qubits as $R$. A constructor for $T$ on $Q$ is some subsystem of $R$ enabling $T$, without undergoing any net change in its ability to do it again. A task is possible if there is no physical constraint on the accuracy to which a constructor can perform it, and impossible otherwise.

Constructor-based irreversibility is defined as the fact that, while $T$ is possible, its transpose $T^{\sim}$ is not. Note that thermodynamics second law can be regarded as requiring this to be true, for some tasks: this is a long-standing tradition, initiated by Planck [15] and continuing with axiomatic thermodynamics $[16,17]$. However, here we will focus on a more general case.

We express now the conditions allowing for a constructor for $T$ under our unitary quantum model. A unitary transformation acting on $Q$ and $R$ will be denoted by $\mathcal{U}_{Q, R}$. For a fixed task $T$ on $Q$ and an $\epsilon>0$, define the set of quantum states of $R$ that can perform $T$ to accuracy $1-\epsilon$ :

$V_{\epsilon}[T] \doteq\left\{\rho_{R}: \mathcal{U}_{Q, R}\left(\rho_{x} \otimes \rho_{R}\right) \mathcal{U}_{Q, R}^{\dagger}=\rho, \operatorname{Tr}_{R}[\rho] \in \epsilon\left(\rho_{y}\right)\right\}$

being $\epsilon\left(\rho_{y}\right)$ the $\epsilon$ ball centered around T's desired output state, $\rho_{y}: \epsilon\left(\rho_{y}\right) \doteq\left\{\sigma: F\left(\rho_{y}, \sigma\right) \geq 1-\epsilon\right\}$, and $F\left(\rho_{\alpha}, \rho_{\beta}\right)=$ $\left[\operatorname{Tr}\left(\sqrt{\sqrt{\rho_{\alpha}} \rho_{\beta} \sqrt{\rho_{\alpha}}}\right)\right]^{2}$ the quantum fidelity [18]. We shall denote with $\mathcal{E}[T]$ a set of $N$ qubits prepared in a state belonging to $V_{\epsilon}$, i.e., a "machine" capable of performing $T$ with error $\epsilon$.

Let us introduce a measure of how much a given $\mathcal{E}[T]$ can perform $T$ to accuracy $1-\epsilon$ after $n$ repeated usages on $n$ fresh substrates $Q_{1}, \ldots, Q_{n}$ initialized as $\rho_{x}^{(n)}=\rho_{x} \forall n$.

We define, for a given initial $\rho_{R}$ state, a recursive expression for the rest state after the $n$th usage of $\mathcal{E}[T]$ :

$$
\begin{aligned}
\rho_{R}^{(1)} & =\operatorname{Tr}_{Q_{1}}\left[\mathcal{U}_{Q_{1}, R}\left(\rho_{x}^{(1)} \otimes \rho_{R}\right) \mathcal{U}_{Q_{1}, R}^{\dagger}\right] \\
& \vdots \\
\rho_{R}^{(n)} & =\operatorname{Tr}_{Q_{n}}\left[\mathcal{U}_{Q_{n}, R}\left(\rho_{x}^{(n)} \otimes \rho_{R}^{(n-1)}\right) \mathcal{U}_{Q_{n}, R}^{\dagger}\right],
\end{aligned}
$$

where $\mathcal{U}_{Q_{n}, R}=U_{Q_{n}, N} \cdots U_{Q_{n}, 1}$ denotes a sequence of unitary interactions between the $n$th substrate qubit and the $N$ rest qubits emerging from the $(n-1)$ th task execution.

The worst-case-scenario steadiness of $\mathcal{E}[T]$ after $n$ usages is defined as

$$
S_{\mathcal{E}[T]}(n) \doteq \operatorname{Inf}_{\rho_{R} \in V_{\epsilon}[T]}\left\{F\left(\rho_{R}, \rho_{R}^{(n)}\right)\right\} .
$$

Most machines lose the ability to perform their task with repeated use, so we expect $S_{\mathcal{E}[T]}(n)$ to decrease with $n$ for a fixed $\epsilon$. A figure of merit for $\mathcal{E}[T]$ resiliency is its relative deterioration after $n$ usages, defined as

$$
\delta_{\mathcal{E}[T]}(n) \doteq \frac{\epsilon}{S_{\mathcal{E}(T)}(n)} .
$$

There are two conditions for a constructor realizing $T$ to be allowed under a given unitary law $\mathcal{U}_{Q_{n}, R}$.

Condition (i). For any arbitrarily small $\epsilon>0$, the set $V_{\epsilon}[T]$ of Eq. (3) is nonempty (i.e., the rest can perform $T$ with arbitrarily high accuracy, once.)

Condition (ii). The relative deterioration $\delta_{\mathcal{E}[T]}(n)$ goes to zero, as $\epsilon$ goes to zero and the number $n$ of repeated usages goes to infinity:

$$
\lim _{\epsilon \rightarrow 0} \lim _{n \rightarrow \infty} \delta_{\mathcal{E}[T]}(n)=0 .
$$

The limits order is relevant for the correct physical interpretation: for a fixed $\epsilon$, we let $n$ go to infinity. This indicates how resilient a machine is.

If both conditions are satisfied, then a sequence of machines $\mathcal{E}[T]$ converges to a limiting machine perfectly retaining the ability to realize $T$ with asymptotically small error, even after $n$ usages, for arbitrarily large $n$ : this limiting machine is a constructor for $T$. A constructor generalizes the notion of catalyst in resource theory [19], with the relaxed requirement to stay within the same set of states instead of the same exact state. Under our assumptions, a constructor being allowed implies that the corresponding task is possible.

Results.-We now demonstrate the compatibility between time-reversal symmetric laws and constructorbased irreversibility with a toy model based on quantum homogenization [20,21] (see Fig. 1), showing that a task $T=\left\{\rho_{x} \rightarrow \rho_{y}\right\}$ being possible does not necessarily imply the same for its transpose $T^{\sim}$. Consider as a homogenization machine the $N$-qubit set $H_{N}[T]$, with each qubit prepared in the state $\rho_{y}$. Suppose that the substrate $Q$, initialized in the state $\rho_{x}$, interacts with the qubits in $H_{N}[T]$ one at a time via the unitary transformation

$$
U_{Q, k}=\cos (\eta) I+i \sin (\eta) \Sigma_{Q k},
$$

where $\Sigma_{Q k}$ is the SWAP gate acting on $Q$ and the $k$ th qubit in $H_{N}[T]$, with $I$ the identity. The SWAP is defined as the gate 


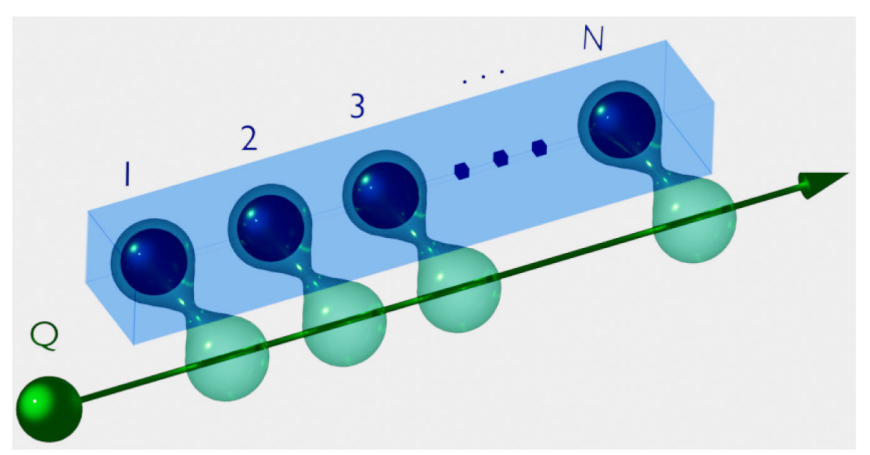

FIG. 1. Qubit-based homogenizer. A substrate qubit $Q$ (in green) interacts with a homogenization machine (azure shaded box) composed of $N$ qubits (in blue). The hourglasses represent the unitary partial swaps $U_{Q, k}(k=1, \ldots, N)$.

$\Sigma_{12}: \Sigma_{12}|\psi\rangle|\phi\rangle=|\phi\rangle|\psi\rangle, \forall|\psi\rangle,|\phi\rangle$ (see, e.g., Ref. [22]). This $U_{Q, k}$ is a partial swap, whose intensity depends on the real parameter $\eta$ : the closer $\eta$ is to $\pi / 2$, the $\operatorname{closer} U_{Q, k}$ is to a standard swAP. For small $\eta$ values, it is a way of slightly modifying the original state of $Q$, making it closer and closer to the target state $\rho_{y}$. The quantum homogenizer is a quantum information formalization of the well-known collision models [23-27]. These models, as well as other open-system dynamics models, see, e.g., Refs. [12,28-31], express irreversibility as emerging from statistical mechanics approximations (such as weak coupling or Markovianity) when the system of interest evolves through the interaction with an environment, typically much larger than the system itself. Here we explain irreversibility without statistical assumptions, considering instead what transformations are possible, and to what accuracy, via quantum homogenization. The state of $Q$ after interacting with $H_{N}[T]$ is

$\rho_{Q, N}=\operatorname{Tr}_{1 \ldots N}\left[\tilde{\prod}_{k=N}^{1} U_{Q, k}\left(\rho_{x} \otimes \rho_{y}^{\otimes N}\right) \tilde{\prod}_{k=1}^{N} U_{Q, k}^{\dagger}\right]$,

where $\tilde{\Pi}$ denotes an ordered product.

Define now the error in performing the task on $Q$ as

$$
\epsilon_{N}=1-F\left(\rho_{Q, N}, \rho_{y}\right) .
$$

As the number of qubits in $H_{N}[T]$ goes to infinity, one has $[20,21]$

$$
\lim _{N \rightarrow \infty} \epsilon_{N}=0,
$$

meaning that the machine $H_{N}[T]$ is capable of performing $T$ perfectly for large $N$, thus satisfying condition (i). This holds for any task $T$ transforming $\rho_{x}$ into $\rho_{y}$. Crucially, however, not all homogenization machines satisfy condition (ii), thus failing to be constructors. Hence, not all tasks are necessarily possible. Specifically, $T^{\sim}$ need not be possible, even if $T$ is possible: this is how constructor-based irreversibility emerges, being radically different from the standard irreversibility of the homogenizer, analyzed in Refs. [20,21] and demonstrated by various qubit implementations (see, e.g., Ref. [32]).

Consider the special case where $\rho_{x}$ and $\rho_{y}$ are, respectively, a pure and a maximally mixed state. In this case, task $T$ goes in the direction of more mixedness, while $T^{\sim}$ does the opposite, purifying the state. For small $\eta$, it is possible to show two facts (see Supplemental Material [33] for details): (i) As $N$ increases, the homogenization machine $H_{N}[T]$ tends to be a constructor for $T$, because its relative deterioration goes to 0 :

$$
\lim _{N \rightarrow \infty} \lim _{n \rightarrow \infty} \delta_{H_{N}[T]}(n)=0 .
$$

(ii) The optimal candidate to perform $T^{\sim}, H_{N}\left[T^{\sim}\right]$, is not a constructor for $T^{\sim}$. Specifically, one can show that

$$
\lim _{N \rightarrow \infty} \lim _{n \rightarrow \infty} \delta_{H_{N}\left[T^{\sim}\right]}(n) \rightarrow \infty .
$$

Thus, $T$ being possible and the assumption of timereversal symmetric laws do not imply that $T^{\sim}$ must also be possible. This makes constructor-based irreversibility compatible with time-reversal symmetric laws under unitary quantum theory.

The experiment.-We provide experimental evidence of this model at work, quantifying the relative deterioration of the homogenizers for $T$ and $T^{\sim}$ and demonstrating the emergence of constructor-based irreversibility within a unitary quantum framework. Initially, we verified experimentally that the homogenizer performing the task $T=$ $\rho_{p} \rightarrow \rho_{m}$ (being $\rho_{p}$ a pure state and $\rho_{m}$ a mixed one) is more efficient than the one performing the transpose task $T^{\sim}=\rho_{m} \rightarrow \rho_{p}$, even at the first task execution $(n=1)$. Consider two states: the pure state $\rho_{p}=|0\rangle\langle 0|$ and the quasimaximally mixed state $\rho_{m}=((1+\gamma) / 2)|0\rangle\langle 0|+$ $((1-\gamma) / 2)|1\rangle\langle 1|$ (with $\gamma \ll 1$ accounting for experimental imperfections in the mixture preparation). We compare the performance of the homogenizers $H_{N}[T]$ and $H_{N}\left[T^{\sim}\right]$ by measuring the error $\epsilon$ of each machine in performing its task, with an experiment (Fig. 2) exploiting single-photon qubits at $1550 \mathrm{~nm}$ (see Supplemental Material [33] for details). Figure 3 shows the results obtained for a partial swap parameter $\eta=(\pi / 4)$.

Respectively, plots (a) and (b) show, for $n=1$, the progression of tasks $T$ and $T^{\sim}$ as the substrate $Q$ interacts with the $k=1, \ldots, N$ qubits of the corresponding homogenizer ( $N=3$ in our setup). The reconstructed diagonal elements $\rho_{00}$ and $\rho_{11}$ of the $Q$ density matrix are reported (by construction, in our case $\rho_{01}=\rho_{10}=0$ ), considering for $T$ the substrate in the initial state $\rho_{p}=|0\rangle\langle 0|$ and the homogenizer qubits in the mixed state $\rho_{m}=0.55|0\rangle\langle 0|+$ $0.45|1\rangle\langle 1|$, and vice versa for $T^{\sim}$. Plot (c) shows the error $\epsilon$ in Eq. (10), indicating the discrepancy between the 


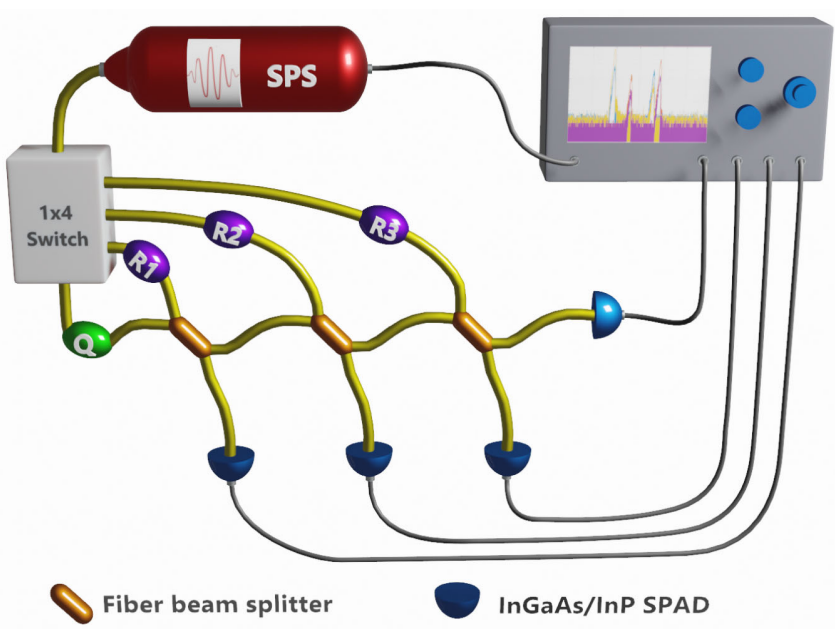

FIG. 2. Experimental setup. Heralded single photons at $1550 \mathrm{~nm}$ are produced by a low-noise single-photon source $[34,35]$ and sent to a $1 \times 4$ fiber optical switch, addressing them either to the substrate path $(Q)$ or to one of our 3-qubit homogenizer paths $(R 1, R 2, R 3)$. The single photons then meet a cascade of $N=3$ fiber beam splitters, each implementing one of the partial swaps realizing the interaction between substrate and homogenizer. Finally, the photons are detected by freerunning infrared detectors, whose output is sent to time-tagging electronics together with the heralding counts.

substrate and target state. Our results clearly show how the homogenizer for $T$ always outperforms the one for $T^{\sim}$. We then extended our analysis to the general case $n>1$, to verify the behavior of the relative deteriorations $\delta_{H_{N}[T]}(n)$ and $\delta_{H_{N}\left[T^{\sim}\right]}(n)$ [see Eq. (6), and Supplemental Material [33] for details]. To this end, we evaluated the machines steadiness with a recursive method considering, for the $j$ th substrate state $Q_{j}(j=1, \ldots, n)$, the machine initialized in a state as close as possible to the one of the machine outgoing the $(j-1)$ th usage. For each $j$, after executing the task and tracing over $Q_{j}$ in Eq. (4), the rest state $\rho_{R}^{(j)}$ presents some entanglement. Not being able to directly measure it, we could nevertheless observe the resulting correlations among the rest qubits, so we reconstructed these correlations (in the computational basis $\{|0\rangle,|1\rangle\}$ ) and reproduced them while initializing the machine state for the $(j+1)$ th usage.

The results for both $T$ and $T^{\sim}$ are reported in Fig. 4, for two experimental $\eta$ values corresponding to a weak interaction between substrate and homogenizer. Here, the relative deteriorations $\delta_{H_{N}[T]}(n)$ and $\delta_{H_{N}\left[T^{\sim}\right]}(n)$ are shown, providing evidence of their asymptotic limits reported in Eqs. (12) and (13). By performing numerical simulations, we studied their behavior for a limited number of $N$ rest qubits and $n$ machine usages, extending our analysis beyond the $N=3$ experimental limit. We investigated both the ideal scenario, with the homogenizer qubits in an entangled state for $n>1$ (solid curves), and the case of (a)

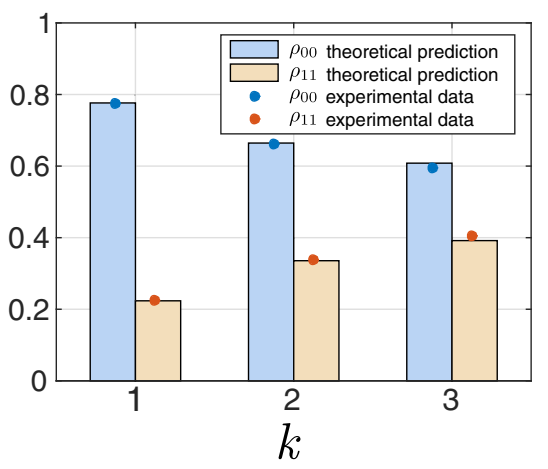

(b)

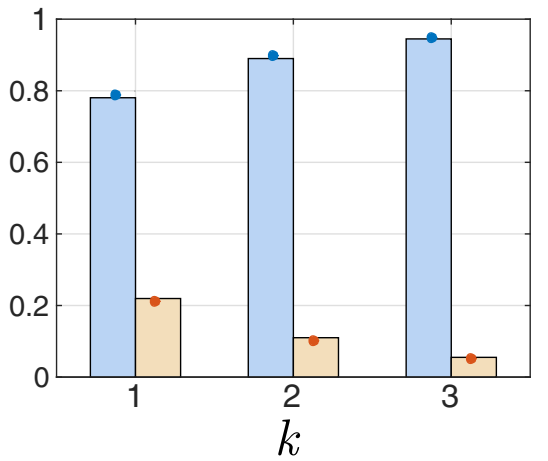

(c)

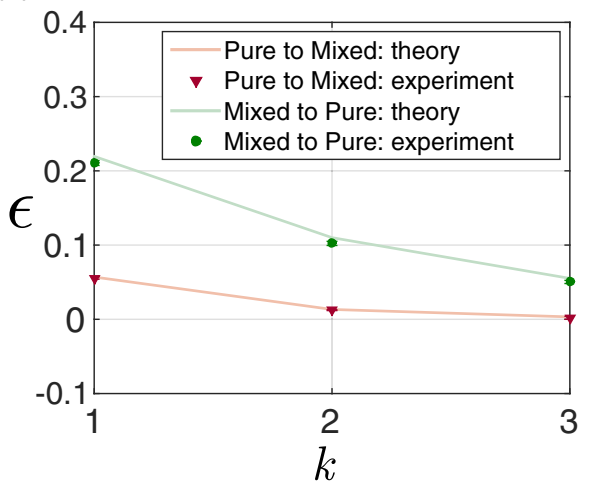

FIG. 3. Results obtained with partial swap parameter $\eta=(\pi / 4)$, for the first usage of the homogenizer $(n=1)$. Panel (a): pure-to-mixed task $T$. The plot shows the $\rho_{00}$ (in azure and blue) and $\rho_{11}$ (in yellow and red) elements of the substrate $Q$ density matrix, initially in the pure state $\rho_{p}=|0\rangle\langle 0|$, after each partial swap $U_{Q, k}$ with one of the $N=3$ homogenizer qubits in the mixed state $\rho_{m}=0.55|0\rangle\langle 0|+0.45| 1\rangle\langle 1|$ (experimentally measured), showing the substrate state evolution induced by the homogenizer. Bars: theoretical predictions; dots: experimentally reconstructed values, reported with the associated uncertainties (within dots size). Panel (b): mixed-to-pure task $T^{\sim}$, being the substrate initial state $\rho_{m}=0.55|0\rangle\langle 0|+0.45| 1\rangle\langle 1|$ and the homogenizer qubits state $\rho_{p}=|0\rangle\langle 0|$. Panel (c): error $\epsilon$ for both $T$ (in orange) and $T^{\sim}$ (in green). Lines: theoretical expectations. Dots: experimental values, with associated uncertainties (within dots size).

the rest qubits forming a separable state for each usage of the homogenizer (dashed curves). For the sake of completeness, we also considered the approximation (dotted curves) of small swap intensity $\eta$ and a homogenizer 

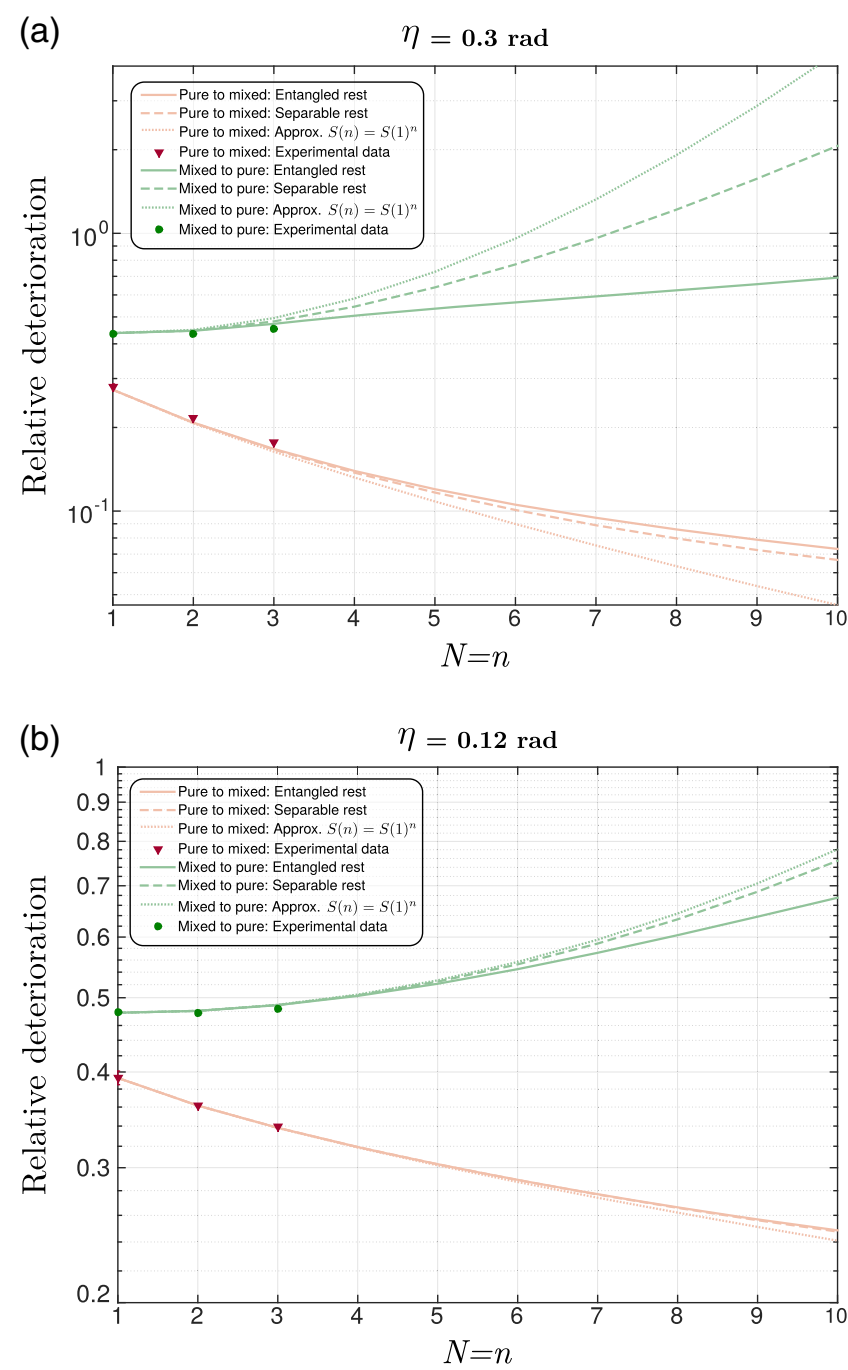

FIG. 4. Constructor-based irreversibility demonstration. Plots (a) and (b) show, for $\eta=0.3$ and $\eta=0.12$, respectively, the relative deterioration of the homogenizer for $T$ and $T^{\sim}$, as a function of the number of machine qubits $N$ and usages $n$. Solid curves: theoretical predictions, considering the rest entangled for $n>1$. Dashed curves: predictions obtained by approximating the rest, for $n>1$, as an ensemble of qubits in a separable state. Dotted curves: approximation considering for each $j=1, \ldots, n$ a separable rest state, in the limit of small $\eta$ and with $S_{\mathcal{E}[T]}(n) \approx S_{\mathcal{E}[T]}(1)^{n}$. Triangles (dots): experimental values obtained for $T\left(T^{\sim}\right)$, with the experimental uncertainty bars below the data points size.

steadiness of the form $S_{\mathcal{E}[T]}(n) \approx S_{\mathcal{E}[T]}(1)^{n}$, with $\rho_{R}^{(n)}$ taken as the tensor product of the reduced density operators of the rest qubits after the $n$th usage. Such approximation allows finding analytical solutions for $\delta_{H_{N}[T]}(n)$ and $\delta_{H_{N}\left[T^{\sim}\right]}(n)$, satisfying the conditions in Eqs. (12) and (13), respectively (details in Supplemental Material [33]).

Figure 4 shows that, for task $T$, the relative deterioration $\delta_{H_{N}[T]}(n)$ steadily decreases for growing $n, N$, qualifying $H_{N}[T]$ as a proper constructor for $T$, according to Eq. (12).
Conversely, we observe that $\delta_{H_{N}\left[T^{\sim}\right]}(n)$ diverges for increasing $n, N$, in agreement with Eq. (13), hence $H_{N}\left[T^{\sim}\right]$ fails to be a constructor for $T^{\sim}$. We can conclude that, in the constructor theory framework, the task $T$ is "possible," while its counterpart $T^{\sim}$ need not be: this is what makes the process corresponding to the task $T$ potentially irreversible.

Conclusions.-We proposed a novel take on the old-age problem of reconciling irreversibility with reversible unitary dynamics with a radically different approach, considering the irreversibility based on tasks possibility or impossibility rather than on statistical considerations about dynamical trajectories being permitted or disallowed. This irreversibility extends the thermodynamical one to a general information-theoretic scenario, thus representing a significant contribution to the development of a generalized quantum thermodynamics [7,36-39]. We used the constructor theory framework, stating that a certain task $T$ being possible does not imply the same for its transpose $T^{\sim}$. Here, we illustrated this idea with a specific example, providing experimental evidence of this mechanism at work. Our experimental results demonstrate that the homogenizer implementing $T$ always outperforms its counterpart for the reverse task $T^{\sim}$, and that the machine for $T^{\sim}$ suffers a much higher degradation than the one realizing $T$, ultimately not satisfying condition (ii) and thus failing to be a constructor. This gives a clear proof of the compatibility of constructor-based irreversibility with unitary quantum theory, providing a frame for the emergence of thermodynamical irreversibility in quantum mechanics [40-45]. In perspective, our work prompts us to seek new interpretations of existing entropy measures by studying the connection of this irreversibility definition with other ones, e.g., in quantum and classical statistical mechanics.

We are grateful to David Deutsch, Benjamin Yadin, Paul Raymond-Robichaud and Maria Violaris for useful discussions. V. V. thanks the Oxford Martin School, the John Templeton Foundation, the EPSRC (UK). E. B. thanks Riccardo Roma for the enlightening discussions, and L. T. K. and F. P. thank Margot Gramegna for helping with the experimental setup optimization. This work was supported by EMPIR projects 17FUN06 "SIQUST", 20FUN05 "SEQUME" and 20IND05 "QADeT" (these projects have received funding from the EMPIR programme co-financed by the Participating States and from the European Union's Horizon 2020 research and innovation programme), and by the European Union's Horizon 2020 FET-OPEN project Grant No. 828946 "Pathos". This research was supported by the Grant No. (FQXi FFF Grant No. FQXi-RFP-1812) from the Foundational Questions Institute and Fetzer Franklin Fund, a donor advised fund of Silicon Valley Community Foundation. This research was also supported by the National Research Foundation, Prime Minister's Office, Singapore, under its Competitive Research Programme (CRP Award No. NRF-CRP14-2014-02) and 
administered by Centre for Quantum Technologies, National University of Singapore. C. M. was also supported by the Templeton World Charity Foundation and by the Eutopia Foundation.

[1] M. Baylin, A Survey of Thermodynamics (AIP Press, New York, 1994).

[2] H. A. Buchdahl, The Concepts of Classical Thermodynamics (Cambridge University Press, Cambridge, England, 1966).

[3] D. Wallace, The quantitative content of statistical mechanics, Stud. Hist. Phil. Mod. Phys. 52, 285 (2015).

[4] R. Landauer, The physical nature of information, Phys. Lett. A 217, 188 (1996).

[5] C. H. Bennett, Demons, engines and the second law, Sci. Am. 257, 108 (1987).

[6] J. Earman and J. D. Norton, Exorcist XIV: The wrath of Maxwell's demon. Part II, Stud. Hist. Phil. Mod. Phys. 30, 1 (1999).

[7] J. Goold, M. Huber, A. Riera, L. del Rio, and P. Skrzypczyk, The role of quantum information in thermodynamics-A topical review, J. Phys. A 49, 143001 (2016).

[8] R. Alicki and R. Kosloff, Introduction to quantum thermodynamics: History and prospects, In Thermodynamics in the Quantum Regime, edited by F. Binder, L. Correa, C. Gogolin, J. Anders, and G. Adesso, Fundamental Theories of Physics vol. 195 (Springer, Cham, 2018).

[9] J. Gemmer, M. Michel, and G. Mahler, Quantum Thermodynamics (Berlin, Springer, 2009).

[10] D. Deutsch, Constructor theory, Synthese 190, 4331 (2013).

[11] D. Deutsch and C. Marletto, Constructor theory of information, Proc. R. Soc. A 471, 20140540 (2015).

[12] H. P. Breuer and F. Petruccione, The Theory of Open Quantum Systems (Claredon Press, Oxford, 2006).

[13] M. Perarnau-Llobet, E. Bäumer, K. V. Hovhannisyan, M. Huber, and A. Acin, No-Go Theorem for the Characterization of Work Fluctuations in Coherent Quantum Systems, Phys. Rev. Lett. 118, 070601 (2017).

[14] P. Talkner, E. Lutz, and P. Hänggi, Fluctuation theorems: Work is not an observable, Phys. Rev. E 75, 050102(R) (2007).

[15] J. Uffink, Bluff your way in the second law of thermodynamics. Stud. Hist. Phil. Mod. Phys. 32, 305 (2001).

[16] C. Carathéodory, Untersuchungen über die Grundlagen der Thermodynamik, Math. Ann. 67, 355 (1909).

[17] E. H. Lieb and J. Yngvason, The physics and mathematics of the second law of thermodynamics, Phys. Rep. 310, 1 (1999).

[18] A. Gilchrist, N. K. Langford, and M. A. Nielsen, Distance measures to compare real and ideal quantum processes, Phys. Rev. A 71, 062310 (2005).

[19] B. Coecke, T. Fritz, and R. Spekkens, A mathematical theory of resources, Inf. Comp. 250, 59 (2016).

[20] M. Ziman, P. Štelmachovič, V. Bužek, M. Hillery, V. Scarani, and N. Gisin, Diluting quantum information: An analysis of information transfer in system-reservoir interactions, Phys. Rev. A 65, 042105 (2002).
[21] V. Scarani, M. Ziman, Štelmachovič, N. Gisin, and V. Bužek, Thermalizing Quantum Machines: Dissipation and Entanglement, Phys. Rev. Lett. 88, 097905 (2002).

[22] F. Vatan and C. Williams, Optimal quantum circuits for general two-qubit gates, Phys. Rev. A 69, 032315 (2004).

[23] T. A. Brun, A simple model of quantum trajectories, Am. J. Phys. 70, 719 (2002).

[24] F. Ciccarello, G. M. Palma, and V. Giovannetti, Collisionmodel-based approach to non-Markovian quantum dynamics, Phys. Rev. A 87, 040103(R) (2013).

[25] J. Gross, C. Caves, G. Milburn, and J. Combes, Qubit models of weak continuous measurements: Markovian conditional and open-system dynamics, Quantum Sci. Technol. 3, 024005 (2018).

[26] F. Ciccarello, S. Lorenzo, V. Giovannetti, and G. M. Palma, Quantum collision models: Open system dynamics from repeated interactions, arXiv:2106.11974.

[27] M. Cattaneo, G. De Chiara, S. Maniscalco, R. Zambrini, and G. L. Giorgi, Collision Models can Efficiently Simulate any Multipartite Markovian Quantum Dynamics, Phys. Rev. Lett. 126, 130403 (2021).

[28] A. O. Caldeira, A. H. Castro-Neto, and T. Oliveira de Carvalho, Dissipative quantum systems modelled by a two-level reservoir coupling, Phys. Rev. B 48, 13974 (1993).

[29] A. Cazzaniga, S. Maniscalco, S. Olivares, and M. G. A. Paris, Dynamical paths and universality in continuousvariable open systems, Phys. Rev. A 88, 032121 (2013).

[30] G. Lindblad, On the generators of quantum dynamical semigroups, Commun. Math. Phys. 48, 119 (1976).

[31] V. Gorini, A. Kossakowski, and E. C. G. Sudarshan, Completely positive dynamical semigroups of N-level systems, J. Math. Phys. (N.Y.) 17, 821 (1976).

[32] M. Violaris, G. Bhole, J. Jones, V. Vedral, and C. Marletto, Transforming pure and mixed states using an NMR quantum homogeniser, Phys. Rev. A 103, 022414 (2021).

[33] See Supplemental Material at http://link.aps.org/ supplemental/10.1103/PhysRevLett.128.080401 for details on the experimental setup, on the partial swap gate and on the derivation of constructor-based irreversibility for the specific case of the quantum homogenizer.

[34] G. Brida, I. P. Degiovanni, M. Genovese, A. Migdall, F. Piacentini, S. V. Polyakov, and I. Ruo Berchera, Experimental realization of a low-noise heralded single-photon source. Opt. Express 19, 1484 (2011).

[35] G. Brida, I. P. Degiovanni, M. Genovese, F. Piacentini, P. Traina, A. Della Frera, A. Tosi, A. Bahgat Shehata, C. Scarcella, A. Gulinatti, M. Ghioni, S. V. Polyakov, A. Migdall, and A. Giudice, An extremely low-noise heralded single-photon source: A breakthrough for quantum technologies, Appl. Phys. Lett. 101, 221112 (2012).

[36] B. Yadin, B. Morris, and G. Adesso, Mixing indistinguishable systems leads to a quantum Gibbs paradox, Nat. Commun. 12, 1471 (2021).

[37] M. Horodecki and J. Oppenheim, Fundamental limitations for quantum and nanoscale thermodynamics, Nat. Commun. 4, 2059 (2013). 
[38] S. Vinjanampathy and J. Anders, Quantum thermodynamics, Contemp. Phys. 57, 545 (2016).

[39] N. Erez, G. Gordon, M. Nest, and G. Kurizki, Thermodynamic control by frequent quantum measurements, Nature (London) 452, 724 (2008).

[40] A. Ghosh, W. Niedenzu, V. Mukherjee, and G. Kurizki, Thermodynamic principles and implementations of quantum machines, in Thermodynamics in the Quantum Regime, edited by F. Binder, L. Correa, C. Gogolin, J. Anders, and G. Adesso, Fundamental Theories of Physics vol. 195 (Springer International Publishing, New York, 2019).

[41] R. Uzdin, Coherence-Induced Reversibility and Collective Operation of Quantum Heat Machines via Coherence Recycling, Phys. Rev. Applied 6, 024004 (2016).
[42] M. Lostaglio, D. Jennings, and T. Rudolph, Description of quantum coherence in thermodynamic processes requires constraints beyond free energy, Nat. Commun. 6, 6383 (2015).

[43] P. M. Riechers and M. Gu, Initial-state dependence of thermodynamic dissipation for any quantum process. Phys. Rev. E 103, 042145 (2021).

[44] V. Scarani, Entanglement and irreversibility in the approach to thermal equilibrium-Known and new results on thermalizing quantum channels for qubits, Eur. Phys. J. Special Topics 151, 41 (2007).

[45] G. Manzano, D. Subero, O. Maillet, R. Fazio, J. P. Pekola, and É. Roldán, Thermodynamics of Gambling Demons, Phys. Rev. Lett. 126, 080603 (2021). 